\title{
APLICAÇÕES DA TOMOGRAFIA DE RESSONÂNCIA MAGNÉTICA NUCLEAR COMO MÉTODO NÃO-DESTRUTIVO PARA AVALIAR OS EFEITOS DE INJÚRIAS MECÂNICAS EM GOIABAS 'PALUMA' E 'PEDRO SATO'
}

\author{
BEN-HUR MATTIUZ ${ }^{2}$, CLOVIS ISBERTO BISCEGLI ${ }^{3}$, JOSÉ FERNANDO DURIGAN ${ }^{2}$
}

\begin{abstract}
RESUMO - Objetivou-se determinar o potencial do uso da tomografia de ressonância magnética, como método não-destrutivo, para avaliar os efeitos das injúrias mecânicas em goiabas. Foram utilizados frutos no estádio de maturação “de vez” das cultivares Paluma e Pedro Sato. Na injúria por impacto, os frutos foram deixados cair, em queda livre, de uma altura de 1,20 m, sofrendo dois impactos, em lados opostos de sua porção equatorial. Na injúria por compressão, os frutos foram submetidos a um peso de 29,4 N, por 15 minutos. Para a injúria por corte, foram efetuados dois cortes, no sentido longitudinal dos frutos, de exatamente $30 \mathrm{~mm}$ de comprimento por $2 \mathrm{~mm}$ de profundidade. Os frutos injuriados foram armazenados sob condições de ambiente $\left(22 \pm 2{ }^{\circ} \mathrm{C}\right.$ e 40 \%UR). Foram realizadas análises com tomógrafo de ressonância magnética Varian Inova de 2 Tesla. As imagens foram obtidas a partir da detecção dos prótons de hidrogênio $\left({ }^{1} \mathrm{H}\right)$. Para cada fruto, foram obtidos tomogramas simétricos a partir do centro do fruto. A tomografia de ressonância magnética nuclear mostrou-se uma ferramenta eficaz na detecção de injúrias internas de frutos. O estresse físico causado pelo impacto produziu um colapso interno nos lóculos desses frutos (internal bruising), levando à perda da integridade celular e a conseqüente liquefação dos tecidos placentários. A cultivar Pedro Sato mostrou uma suscetibilidade maior à injúria por impacto que a 'Paluma'. A injúria por compressão tornou-se mais evidente no pericarpo externo do fruto, de ambas as cultivares. A injúria por corte levou a lignificação dos tecidos no local injuriado e deformações superficiais devido à perda acentuada de matéria fresca no local da lesão, evidentes no sexto dia de avaliação.
\end{abstract}

Termos para indexação: Psidium guajava, pós-colheita, injúrias mecânicas, tomografia, ressonância magnética nuclear, injúria interna.

\section{APPLICATIONS OF THE NUCLEAR MAGNETIC RESONANCE TOMOGRAPHY AS A NON-DESTRUCTIVE METHOD TO EVALUATE THE EFFECTS OF MECHANICAL INJURIES IN 'PALUMA' AND 'PEDRO SATO' GUAVAS}

\begin{abstract}
The present study reports on the potential of the use of the tomography of magnetic resonance, as a non-destructive method, to evaluate the effects of the mechanical injuries in guava fruits. Fruits were harvested at mature stage of Paluma and Pedro Sato cultivars. In impact injury, fruits were allowed to fall freely from a height of $1.2 \mathrm{~m}$, subjecting them to two impacts on opposites sides of the equatorial portion of the fruit. In compression injury, fruits were submitted to a weight of $29.4 \mathrm{~N}$ for $15 \mathrm{~min}$. For the cut injury, two longitudinal cuts were made, $30 \mathrm{~mm}$ in length and $2 \mathrm{~mm}$ deep. Fruits were stored under ambient conditions $\left(22 \pm 2{ }^{\circ} \mathrm{C}, 40 \% \mathrm{RH}\right)$. Magnetic resonance images (MRI) of intact guava were acquired using the Varian 2 Tesla tomography. Images were obtained from the hydrogen $\left(\mathrm{H}^{1}\right)$ of the water content and in each fruit was obtained symmetrical image from the center of the fruit. The magnetic resonance image ( MRI) technique was shown to be an effective tool in the detection of internal injuries of fruits. The physical stress caused by the impact produced an internal collapse of fruit locules (internal bruising), taking to the loss of the cellular integrity and the consequent liquefaction of the placentary tissues. The Pedro Sato cultivar showed a larger susceptibility to impact injury that the 'Paluma'. The compression injury became more evident in the external pericarpo of the fruit, for both cultivars. The cut injury took the lignification of the tissues in the injured place and superficial deformations due to the accentuated loss of fresh matter in the place of the lesion, evident in the sixth day of evaluation.
\end{abstract}

Index Terms: Psidium guajava, postharvest, mechanical injuries, NMR tomograph, internal bruising, Magnetic resonance image.

\section{INTRODUÇÃO}

Atualmente, a produção de frutas de alta qualidade, objetivando o comércio de produtos frescos, para mercados cada vez mais exigentes, tem sido a tônica da fruticultura brasileira. Isso se deve às mudanças culturais nos hábitos alimentares do brasileiro, notadamente, nas populações de classe média a média alta (Souza, 2001).

Essas exigências requerem técnicas seguras, rápidas e não destrutivas para a medida de propriedades físicas dos frutos (Thomas et al., 1995; Clark et al., 1997).

A espectroscopia por Ressonância Magnética Nuclear (Magnetic Resonance Spectroscopy, MRS) constitui um método não-invasivo, seguro e capaz de fornecer informações sobre o estado químico e físico dos materiais, bem como sobre o estado fisiológico e as condições de metabolismo em sistemas biológicos, sem qualquer extração ou destruição da amostra (Bottomley, 1982; Clark et al., 1997).

A condição fundamental para analisar-se algum material por MRS é a presença de núcleos com momento magnético. No caso de frutas, o núcleo mais indicado é o do hidrogênio $\left({ }^{1} \mathrm{H}\right)$, que, devido a sua abundância, resulta num alto valor da relação sinal/ruído e, conseqüentemente, permite a obtenção de espectros e imagens em tempos curtos. A mobilidade desses núcleos de hidrogênio nos frutos varia com os pro- cessos metabólicos e maturação. Adicionalmente, essas concentrações e a mobilidade dos átomos de ${ }^{1} \mathrm{H}$ estão associadas com atributos qualitativos desses frutos, como a ocorrência de injúrias mecânicas nos tecidos (Chen et al., 1996).

Considerando que os parâmetros da ressonância magnética da água em alimentos são dependentes de sua arquitetura celular, as mudanças que afetam a sua estrutura podem ser detectadas por tomografia por ressonância magnética através da formação de imagens (Magnetic Resonance Imaging, MRI) (Nascimento et al., 1999; Biscegli et al., 2000).

Estudos preliminares realizados por Chen et al. (1989) indicam a MRI como poderosa ferramenta para fornecer informações sobre a estrutura interna de frutas inteiras, relacionado-as à qualidade, como a ocorrência de injúrias mecânicas, regiões desidratadas, danos por larvas, amolecimento interno e estádio de maturação. Clark \& Burmeister (1999) utilizaram a MRI para identificar injúrias em maçãs, causadas por altas concentrações de $\mathrm{CO} 2$ durante o armazenamento sob condições de atmosfera controlada. Vários autores vêm utilizando a tomografia de ressonância magnética, como método não destrutivo, a fim de avaliar a qualidade de frutas frescas (Zion, et al., 1995; Hall et al., 1998; Biscegli et al., 2000; Gonzales et al., 2001). Chen et al. (1996) e Clark et al. (1999) utilizaram a MRI para acompanhar o desenvolvimento em frutas, através de medidas quantitativas fornecidas pelas imagens.

1 (Trabalho 047/2002). Recebido: 11/03/2002. Aceito para publicação: 25/06/2002.

Parte da Tese de Doutorado apresentada pelo primeiro autor à FCAV-UNESP, Campus de Jaboticabal.

2 Departamento de Tecnologia, FCAV-UNESP, Campus de Jaboticabal. Via de acesso Prof. Paulo Donato Castellane, Km 5, 14870-000. bmattiuz@ig.com.br 3 Pesquisador da EMBRAPA - Instrumentação Agropecuária. Caixa Postal 741, CEP 13560-970 São Carlos-SP 
Objetivou-se determinar o potencial do uso da tomografia de ressonância magnética, através do software de processamento de imagens SIARCSÓ, como método não-destrutivo, para avaliar os efeitos das injúrias mecânicas em goiabas 'Paluma' e 'Pedro Sato'.

\section{MATERIALEMÉTODOS}

Foram utilizados frutos de goiabeiras das cultivares Paluma e Pedro Sato procedentes do município de Vista Alegre do Alto-SP.

Depois de colhidos, no estádio de maturação "de vez", correspondente à coloração verde-mate (Pereira, 1995), os frutos foram imediata e cuidadosamente transportados para o Laboratório de Tecnologia dos Produtos Agrícolas da FCAV/UNESP - Jaboticabal, onde foram inicialmente imersos em água fria $\left(15^{\circ} \mathrm{C}\right)$ e clorada $\left(150 \mathrm{mg}\right.$ de cloro. $\left.\mathrm{L}^{-1}\right)$ por cinco minutos e, em seguida, submetidos às injúrias mecânicas.

$\mathrm{Na}$ injúria por impacto, eles foram deixados cair, em queda livre, de uma altura de $1,20 \mathrm{~m}$. Cada fruto sofreu dois impactos em sua região equatorial, em lados opostos. Para a injúria correspondente à compressão, os frutos foram colocados em um aparelho onde um bloco exercendo um peso de 29,4 $\mathrm{N}$ era apoiado, por 15 minutos, provocando 2 lesões em lados opostos e no sentido longitudinal dos frutos. Na injúria por corte, foram realizados dois cortes, em lados opostos, de $30 \mathrm{~mm}$ de comprimento por $2 \mathrm{~mm}$ de profundidade, no sentido longitudinal, usando-se uma lâmina com 1,1 mm de espessura. As áreas lesionadas eram imediatamente demarcadas.

Em seguida, os frutos foram transportados cuidadosamente até a EMBRAPA Instrumentação Agropecuária, em São Carlos-SP, onde foram armazenados a $22 \pm 2{ }^{\circ} \mathrm{Ce} 40 \% \mathrm{UR}$.

Os frutos foram analisados em tomógrafo de ressonância magnética nuclear Varian Inova de 2 Tesla, com a inserção dos mesmos numa bobina de radiofreqüência do tipo "gaiola", com diâmetro interno de $14 \mathrm{~cm}$, operando na freqüência de $85,53 \mathrm{MHz}$. As imagens foram obtidas a partir da detecção dos prótons de hidrogênio $\left({ }^{1} \mathrm{H}\right)$, que são essencialmente das moléculas de água que compõem as frutas. As imagens geradas são em matrizes de 256 x 256 pixels, em 256 tons de cinza, em fatias com $2 \mathrm{~mm}$ de espessura e espaçadas de $5 \mathrm{~mm}$. Para cada fruto, foram obtidos 9 tomogramas simétricos, a partir do centro do fruto, em cortes sagitais. As imagens bidimensionais foram analisadas com relação à forma, localização e textura dos graus de cinza, que indicam as situações da água, mais móvel (livre) ou mais ligada aos tecidos sadios. Essas imagens foram captadas com intervalos de cinco dias, após a aplicação das injúrias. Foram realizadas imagens do tipo coronal, para as injúrias por impacto e por compressão, e sagital para as injúrias por corte.

\section{RESULTADOSEDISCUSSÃO}

Nas Figuras 1 e 2, são apresentadas as imagens por tomografia de ressonância nuclear magnética de frutos injuriados e não injuriados de goiabas 'Paluma' e 'Pedro Sato'. As imagens obtidas são observadas essencialmente a partir dos sinais dos núcleos de ${ }^{1} \mathrm{H}$ da água, que corresponde a, pelo menos, $93 \%$ da intensidade do espectro de ${ }^{1} \mathrm{H}$ dos frutos de goiaba.

Na parte saudável da fruta, correspondente aos frutos-controle, a diminuição da massa molecular implicou o aumento da mobilidade das moléculas de água, proporcionando aumento do tempo de relaxação spin-spin (T2), resultando numa tonalidade mais clara na imagem. Observa-se que essas áreas mais claras se tornam mais evidentes ao longo do período de armazenamento ( $\left.6^{\circ} \mathrm{dia}\right)$. Provavelmente, essa resposta indica o aumento da concentração de açúcares, devido à hidrólise de carboidratos de reserva e ao colapso da matriz celular, devido ao amadurecimento (Hall et al., 1998).

Por outro lado, áreas mais claras servem também para indicar condições de água móvel (livre), causadas por injúrias de natureza mecânica. Nas Figuras 1 e 2, é possível constatar esse efeito, onde o estresse físico causado pelo impacto produziu um colapso interno nos lóculos desses frutos (internal bruising), levando à perda da integridade celular e à conseqüente liquefação dos tecidos placentários, com extravasamento celular. Isto é perfeitamente visível através das áreas mais claras localizadas no interior dos frutos (indicado por setas). Observa-se ainda que, nas goiabas, a injúria por impacto não apresenta sintomas externos prontamente visíveis, ou seja, o pericarpo externo do fruto permanece aparentemente intacto no momento da injúria e evolui para regiões lesionadas com o passar dos dias. Moretti (1998) também observou a ocorrência de injúria interna em tomates submetidos à injúria mecânica por impacto, verificando que elas nem sempre eram acompanhadas por sintomas externos visíveis. Áreas escurecidas também foram detectadas, através de MRI, em maçãs 'Braeburn', durante o armazenamento em atmosfera controlada sob altas concentrações de $\mathrm{CO}_{2}$ (Clark \& Burmeister, 1999). Essa técnica também permitiu a detecção de distúrbios internos em peras (Wang \& Wang, 1989), maçãs (Wang et al., 1988; Gonzalez et al., 2001), nectarinas (Sonego et al., 1995) e melões (Hall et al., 1998).

A injúria por compressão produziu lesões (áreas mais esbranquiçadas e indicadas por setas) no pericarpo externo dos frutos de ambas as cultivares de goiaba (Figuras 1 e 2). As regiões lesionadas são tão mais brancas quanto mais próximas da casca dos frutos, provavelmente devido à maior proximidade do objeto compressor. Mohsenin (1986) afirma que a resistência de um determinado vegetal depende de suas propriedades reológicas. Torna-se evidente que a estrutura e a elasticidade celular da goiaba conferiu resistência mecânica à compressão, fazendo com que os tecidos mais internos fossem preservados.

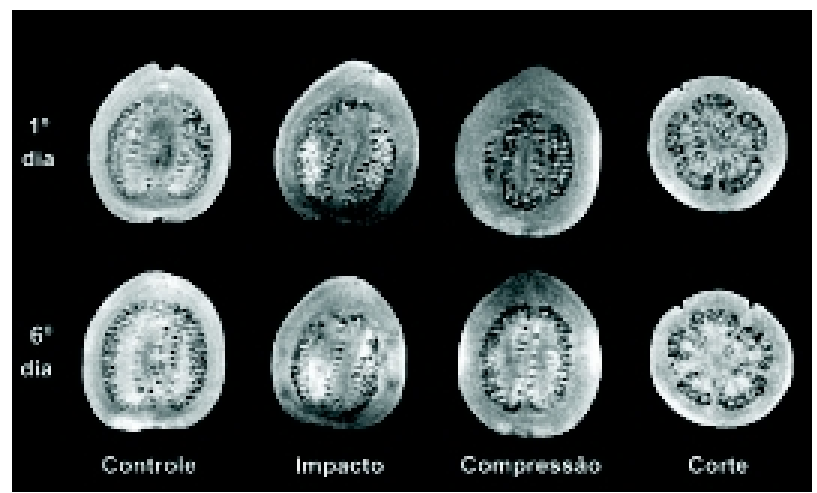

FIGURA 1 - Tomogramas de ressonância magnética nuclear de goiabas 'Paluma' submetidas a três tipos de injúrias mecânicas.

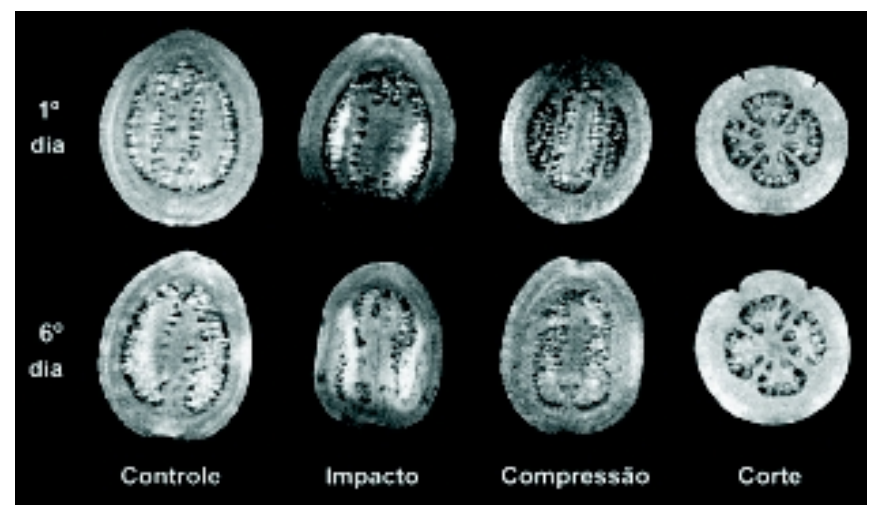

FIGURA 2 - Tomogramas de ressonância magnética nuclear de goiabas 'Pedro Sato' submetidas a três tipos de injúrias mecânicas.

Quando se compara as cultivares em cada injúria, percebe-se que, na injúria por impacto, a cultivar Pedro Sato mostra uma suscetibilidade maior a esse tipo de injúria que a 'Paluma', evidenciada pela maior região esbranquiçada. Esses resultados corroboram com os trabalhos de Mattiuz \& Durigan (2001), que, ao submeterem frutos das cultivares Paluma e Pedro Sato a injúrias mecânicas, evidenciaram que, na injúria por impacto, os frutos da 'Paluma' apresentaram uma firmeza 
significativamente maior (2,85 kPa) que os da 'Pedro Sato' $(2,49 \mathrm{kPa})$.

As Figuras 1 e 2 também permitem visualizar que a injúria por corte promoveu a ocorrência de áreas mais esbranquiçadas, indicativo de água livre, nas regiões próximas aos locais dos cortes, no primeiro dia, em ambas as cultivares. No $6^{\circ}$ dia de avaliação, esta área não apresentava sintomas de injúria, provavelmente devido à lignificação dos tecidos nessa região, interrompendo o avanço da injúria. Contudo, verifica-se que, na região vizinha ao corte, houve deformações no formato do fruto, em ambas as cultivares. Isto pode ser devido à perda acentuada de massa fresca no lugar da injúria, ocasionando uma redução na superfície e dando origem à concavidade no local injuriado.

\section{CONCLUSÕES}

1 - A tomografia de ressonância magnética é uma ferramenta eficaz na detecção de injúrias internas de frutos.

2 - O estresse físico causado pelo impacto produziu um colapso interno nos lóculos desses frutos, levando à perda da integridade celular e à conseqüente liquefação dos tecidos placentários.

3 - A injúria por compressão tornou-se mais evidente no pericarpo externo do fruto, mostrando lesões nos tecidos.

4 - A injúria por corte provocou deformações superficiais devido à perda de matéria fresca no local da lesão.

\section{REFERÊNCIASBIBLIOGRÁFICAS}

BISCEGLI, C. I.; FRANCO, R. W. de A.; TANNUS, A.; COLNAGO, L. A Use of magnetic resonance and spectroscopy in tropical fruits: challenges and opportunities. In: CRUVINEL, P. E.; COLNAGO, L. A. (Ed.) Advances in agricultural tomography. São Carlos: EMBRAPA Agricultural Instrumentation, 2000. p. 77-78.

BOTTOMLEY, P.A. NMR imaging techniques and applications: A review. Review of Scientific Instruments, New York, v. 53, n. 9, p. 13191337, 1982.

CANTWELL, M. Postharvest handling systems: Minimally processed fruits and vegetables. In: KADER, A.A. (Ed.) Postharvest technology of horticultural crops. 2. ed. Davis:University of California, 1992. p. 277-281.

CHEN, P.; McCARTHY, M.J.; KAUTEN, R. NMR for Internal Quality Evaluation of Fruits and Vegetables. Transactions of the ASAE, St. Joseph, v. 32, n. 5, p. 1747-1753, 1989.

CHEN, P.; McCARTHY, M.J.; KIM, S.-M.; ZION, B. Development of a high-speed NMR technique for sensing maturity of avocados. Transactions of the ASAE, St. Joseph, v. 39, n. 6, p. 2205-2209, 1996.

CLARK, C.J.; BURMEISTER, D.M. Magnetic resonance imaging of browning development in 'Braeburn' apple during controlled-atmosphere storage under high $\mathrm{CO}_{2}$. HortScience, Alexandria, v. 34, n. 5, p. 915-919, 1999.

CLARK, C.J.; HOCKINGS, P.D.; JOYCE, D.C.; MAZUCCO, R.A. Application of magnetic resonance imaging to pre- and post-harvest studies of fruits and vegetables. Postharvest Biology and Technology,
Amsterdam, n. 11,p. 1-21, 1997.

CLARK, C.J.; RICHARDSON, A.C.; MARSH, K.B. Quantitative magnetic resonance imaging of Satsuma Mandarin fruit during growth. HortScience, Alexandria, v. 34, n. 6, p. 1071-1075, 1999.

GONZALEZ, J.J.; VALLE, R.C.; BOBROFF, S.; BIASI, W.V.; MITCHAM, E.J.; McCARTHY, M.J. Detection and monitoring of internal browning development in 'Fuji' apples using MRI. Postharvest Biology and Technology, Amsterdam, n. 22, p. 179-188, 2001.

HALL, L.D.; EVANS, S.D.; NOTT, K.P. Measurement of textural changes of food by MRI relaxometry. Magnetic Resonance Imaging, Amsterdam, v. 16, n. 5/6, p. 485-492, 1998.

MATTIUZ, B.H.; DURIGAN, J.F. Efeito de injúrias mecânicas na firmeza e coloração de goiabas das cultivares Paluma e Pedro Sato. Revista Brasileira de Fruticultura, Jaboticabal, v. 23, n. 2, p. 277-281, 2001.

MOHSENIN, N.N. Physical properties of plant and animal materials: structure, physical characteristics and mechanical properties. 2. ed. New York: Gordon and Breach, 1986. 891p.

MORETTI, C.L. Injúria interna de impacto em frutos de tomate: fisiologia e conservação pós-colheita. 1998. 132f. Tese (Doutorado em Produção Vegetal) - Universidade Federal de Viçosa, Viçosa, 1998.

NASCIMENTO, A.S. do; BISCEGLI, C.I.; MENDONÇA, M. da C.; CARVALHO, R. da S. Avanços em tratamentos quarentenários para exportação de manga brasileira: tratamento hidrotérmico e tomografia de resonância magnética. In: ALVES, R. E.; VELOZ, C. S. (Org.) Exigências quarentenárias para exportação de frutas tropicais $\mathrm{e}$ subtropicais. Fortaleza: EMBRAPA Agroindústria Tropical/CYTED/ CONACYT, 1999. p.155-171.

PEREIRA, F.M. Cultura da goiabeira. Jaboticabal:FUNEP, 1995. 47p.

SONEGO, L.; BEN-ARIE, R.; RAYNAL, J.; PECH, J.C. Biochemical and physical evaluation of textural characteristics of nectarines exhibiting woolly breakdown: NMR imaging, X-ray computed tomography and pectin composition. Postharvest Biology and Technology, Amsterdam, v. 5, n. 3, p. 187-198, 1995.

SOUZA, R. A. M. de. Mercado para produtos minimamente processados. Informações Econômicas, São Paulo, v. 31, n. 3, p. 7-18, 2001.

THOMAS, P.; KANNAN, A.; DEGWEKAR, V. H.; RAMAMURTHY, M. S. Non-destructive detection of seed weevil-infested mango fruits by X-ray imaging. Postharvest Biology and Technology, Amsterdam, n. 5, p. 161-165, 1995.

WANG, C.Y.; WANG, P.C. Nondestructive detection of core breakdown in 'Barlett' pears with nuclear magnetic resonance imaging. HortScience, Alexandria, v. 24, n. 1, p. 106-109, 1989.

WANG, S.Y.; WANG, P.C.; FAUST, M. Non-destructive detection of watercore in apple with nuclear magnetic resonance imaging. Scientia Horticulturae, Amsterdam, v. 35, p. 227-234, 1988.

WATADA, A.E.; KO, N.P.; MINOTT, D.A. Factors affecting quality of fresh-cut horticultural products. Postharvest Biology and Technology, Amsterdam, n. 9, p. 115-125, 1996.

ZION, B.; CHEN, P.; McCARTHY, M.J. Nondestructive quality evaluation of fresh Prunes by NMR spectroscopy. Journal of the Science of Food \& Agriculture, Sussex, v. 67, p. 423-429, 1995. 